\title{
Orientation of the monomeric porin OmpG in planar lipid bilayers
}

\author{
Dr. Min Chen ${ }^{[a]}$, Qiu-Hong Li[a], and Prof. Hagan Bayley[a] \\ Hagan Bayley: hagan.bayley@chem.ox.ac.uk \\ [a]Department of Chemistry, University of Oxford, 12 Mansfield road, Oxford OX1 3TA, UK, Fax: \\ (+)441865275708
}

\section{Abstract}

Outer membrane protein $\mathrm{G}(\mathrm{OmpG})$ is a non-selective porin from E. coli. OmpG is a monomer, which makes it unusual among porins, and suggests that it may be useful in biotechnology. In planar lipid bilayers, individual OmpG pores reconstituted by insertion from detergent exhibit pronounced asymmetry in current-voltage relationships and voltage-dependent gating. Here, this asymmetry is used to deduce the orientation of OmpG in the bilayers. We introduced two cysteines into the extracellular loops of OmpG. Cleavage of the disulfide bond formed by these residues significantly increases spontaneous gating of the pore. By adding DTT to one side of the bilayer, or the other, we demonstrated that pores showing a quiet trace at negative potentials have a "trans" conformation (extracellular loops on the trans side of the bilayer), while pores showing a quiet trace at positive potentials have a "cis" conformation (extracellular loops on the cis side). With this knowledge, we examined the binding of a cyclodextrin to OmpG. When the cyclodextrin was presented to the extracellular face of the pore, transient multisite interactions were observed. By contrast, when the cyclodextrin was presented to the periplasmic face, a more stable single-site interaction occurred. Because the cyclodextrin can act as a molecular adapter by binding analytes, this information serves to advance the use of OmpG as a biosensor

\section{Keywords}

outer membrane protein G; orientation; biosensor; planar lipid bilayer; cyclodextrin

\section{Introduction}

The planar lipid bilayer (PLB) technique is a powerful and versatile approach for investigating the functional properties of transmembrane channels and pores. ${ }^{[1]}$ Over the last decade, stochastic sensing, a potentially important means of single molecule detection, has been developed, based primarily on single-channel recording in PLBs. ${ }^{[2,3]}$ For stochastic detection, the ionic current passing through an individual protein pore, containing an engineered recognition site, is monitored in the presence of analytes. By this means, information about the identity of the analytes, as well as their concentrations, is gathered. $[2,3]$

So far, PLB recording has been used to characterize the functional properties of a wide variety of proteins including bacterial outer membrane porins, ${ }^{[4-8]}$ mammalian plasma membrane ion channels, ${ }^{[9]}$ mitochondrial proteins ${ }^{[10]}$ and toxins, ${ }^{[11]}$ as well as engineered

Correspondence to: Hagan Bayley, hagan.bay ley@chem.ox.ac.uk.

Supporting information for this article is available on the WWW under http://www.chembiochem.org 
and synthetic channels and pores. ${ }^{[12]}$ For those proteins containing an extramembraneous domain, as well as a transmembrane domain, such as staphylococcal a-hemolysin (aHL) and TolC, ${ }^{[13,14]}$ direct insertion from the solution into the lipid bilayer is governed by the inability of the extramembraneous domain to cross the bilayer and therefore is unidirectional. ${ }^{[15]}$ By comparison, proteins with insubstantial extramembraneous domains, such as porins and certain ion channels, can incorporate into the lipid bilayer in both orientations. [16-18]

Knowledge of the absolute orientation of a transmembrane channel or pore is important for studying the mechanism of gating, interactions with ligands and physiological function. ${ }^{[6,16]}$ Information on orientation is also important for drug screening. ${ }^{[19,20]}$ For example, whether a therapeutic agent exerts its function from the extracellular or intracellular side of a membrane will determine the drug delivery strategy. In addition, when aHL is used as a stochastic sensor, the direction from which the analyte or the adapter molecule $\beta$-cyclodextrin ( $\beta C D$ ) enters the pore lumen affects the way in which the electrical current is modulated. $[21,22]$

Outer membrane protein $\mathrm{G}(\mathrm{OmpG})$ is a monomeric porin from E. coli. ${ }^{[23-25]}$ It is a $14-$ stranded $\beta$ barrel, with long loops at the extracellular end and short turns facing the periplasm. ${ }^{[26-28]}$ Because OmpG is a monomer, it is an attractive alternative to the heptameric aHL pore for engineering as a stochastic sensor. ${ }^{[2]}$ However, wild-type (WT) OmpG exhibits spontaneous gating activity which would interfere with a signal arising from analyte binding. Recently, a mutant form of OmpG, qOmpG, was obtained by using mutagenesis guided by molecular dynamics calculations to eliminate $95 \%$ of the spontaneous gating. ${ }^{[29]}$ qOmpG equipped with a molecular adapter, heptakis-(6-deoxy-6amino)- $\beta$-cyclodextrin $\left(\mathrm{am}_{7} \beta \mathrm{CD}\right)$, was used for the detection of ADP. ${ }^{[29]}$ The determination of the absolute orientation of the OmpG pore in planar lipid bilayers is necessary to advance its development as a biosensor.

In previous work, we have shown that a disulfide bond located in the extracellular loops of an OmpG mutant, OmpG S-S, acts as a molecular switch controlling the gating behavior of the pore (Fig. 1); ${ }^{29]}$ cleavage of the disulfide bond with DTT increases the gating activity. In the present study, we used this feature to determine the absolute orientation of OmpG in a planar lipid bilayer. First, we established a rule to distinguish the two different orientations of OmpG pores according to their asymmetric responses to positive and negative applied potentials. Second, by comparing the response of OmpG S-S pores to DTT presented from either the cis or the trans side of the lipid bilayer, we were able to determine the absolute orientation of individual pores. With this knowledge, we investigated the interactions between the $q O m p G$ pore and the molecular adapter $a_{7} \beta C D$ presented from either the extracellular or periplasmic entrances.

\section{Results and Discussion}

\section{Asymmetric gating patterns and conductance values of wild-type OmpG}

The properties of wild-type (WT) OmpG pores were studied by single-channel current recording. In a planar bilayer, WT OmpG exhibits spontaneous gating at both positive and negative potentials (Fig. 2). The gating probability, $P_{\text {gating }}$, is defined as the time a channel spends in closed or partially closed states divided by the total recording time. The records at positive and negative potentials differ in their gating patterns, and in the example shown (Fig. 2) the traces from negative potentials showed both more gating spikes and higher $P_{\text {gating }}$ values (Fig. 2). Increases in the applied potential enhance the asymmetry of the gating behavior (Fig. 2). For a given pair of positive and negative applied potentials, we define the trace with lower $P_{\text {gating }}$ value as the quite trace $(\mathrm{Q}$-trace) and the other trace as the 
noisy trace (N-trace). In the example (Fig. 2), the Q-trace occurs at all positive potentials except for $+25 \mathrm{mV}$, a potential below which OmpG pores exhibit very weak asymmetry in their gating. However, Q-traces were seen at negative potentials with other OmpG pores (Fig. S1, supporting information (SI)). In these cases, we surmise that the OmpG protein inserted into the lipid bilayer in the opposite direction to that displayed here (Fig. 2). We name the orientation of OmpG pores showing a Q-trace at positive potential and a $\mathrm{N}$-trace at negative potential $\mathrm{Q}^{+} / \mathrm{N}^{-}$; the opposite orientation is $\mathrm{Q}^{-} / \mathrm{N}^{+}$. The ratio of $\mathrm{Q}^{-} / \mathrm{N}^{+}$to $\mathrm{Q}^{+} / \mathrm{N}^{-}$ pores was $6: 4(n=30)$, indicating that the insertion of $O m p G$ pores into planar lipid bilayers under our experimental condition (DPhPC bilayer at an applied potential of +200 $\mathrm{mV}$ ) is bi-directional, with little preference for either orientation.

In addition to the asymmetric gating pattern, the unitary conductance values of the OmpG pore at positive and negative potentials also differ. The currents at negative potentials are larger than those at positive potentials for a $\mathrm{Q}^{+} / \mathrm{N}^{-}$pore (Fig. 2), with a more obvious difference at higher potentials (Fig. 2). For $\mathrm{Q}^{-} / \mathrm{N}^{+}$pores, the higher conductance values occur at positive potentials (Fig. S1). In other words, $\mathrm{N}$-traces always exhibit higher conductance values when compared with the Q-trace at the opposite potential (Fig. 4A). Thus, the relative orientation $\left(\mathrm{Q}^{+} / \mathrm{N}^{-}\right.$or $\left.\mathrm{Q}^{-} / \mathrm{N}^{+}\right)$of a pore can be confirmed by comparing the gating pattern with the unitary conductance values at positive and negative potentials.

\section{Asymmetric gating patterns and conductance values of OmpG S-S and SH-SH}

As previously demonstrated, the mutant OmpG S-S showed less gating activity at $\pm 50 \mathrm{mV}$ compared to WT OmpG. For example, in a typical case, the $P_{\text {gating }}$ values were 0.042 and 0.017 at $-50 \mathrm{mV}$ and $+50 \mathrm{mV}$, respectively (Fig. 3A). ${ }^{[29]}$ After the disulfide bond was cleaved by DTT, the gating activity of the mutant (now called OmpG SH-SH) increased (Fig. 3B). The reduced pore exhibits a gating pattern similar to WT OmpG. The $P_{\text {gating }}$ values of a typical OmpG SH-SH pore were 0.104 and 0.050 at $-50 \mathrm{mV}$ and $+50 \mathrm{mV}$, respectively. Besides the differences in the $P_{\text {gating }}$ values, the gating events of the Q-traces in S-S and SH-SH differ significantly in the amplitudes of the current blockades (Fig. 3C). The event amplitude histograms reveal that the gating events of S-S are located in a lower range than those of SH-SH. In S-S, the major peak appeared at $\sim 14 \mathrm{pA}$ at $+50 \mathrm{mV}$ and $\sim 25$ $\mathrm{pA}$ at $+125 \mathrm{mV}$. By contrast, in SH-SH, the major peak appeared at $\sim 28 \mathrm{pA}$ at $+50 \mathrm{mV}$ and $\sim 55 \mathrm{pA}$ at $+125 \mathrm{mV}$ (Fig. 3C).

Like WT OmpG, the N-traces of mutants S-S and SH-SH were associated with higher unitary conductance values than those of the Q-traces (Fig. 4B, C). Also, increasing the applied voltage enhanced the asymmetry of the pore gating. These data indicate that the mutations did not change the overall pattern of asymmetrical behavior of the OmpG pore.

\section{Determination of the orientation of OmpG S-S by its response to DTT added from either the cis or trans side of the bilayer}

In all experiments, OmpG protein was added to the cis chamber. The insertion of a single OmpG pore can occur in two different ways: (I): The periplasmic turns pass through the bilayer, leaving the extracellular loops located at the cis surface and the protein in the "cis" orientation; (II): The extracellular loops pass through the bilayer and end up at the trans surface, with the protein in the "trans" orientation (Fig. 5A). In the case of OmpG S-S, the disulfide bond of the cis orientation will be readily cleaved by the addition of DTT to the cis chamber. However, to cleave the disulfide bond of the trans orientation, cis DTT must pass through the pore or the bilayer. This eventuality was ruled out by adding DTT to one chamber and $\mathrm{H}_{2} \mathrm{O}_{2}$ to the other, because DTT that crossed the bilayer was destroyed in the opposing chamber. 
The cleavage of the disulfide bond in OmpG S-S alters the gating pattern (Fig. 3). Taking advantage of this, the proximity of the disulfide bond in OmpG S-S to the bilayer surface was examined by adding DTT or $\mathrm{H}_{2} \mathrm{O}_{2}$ to the chambers, selected as described below. Each pore was recorded for $5 \mathrm{~min}$ at both positive and negative potentials to determine its relative orientation $\left(\mathrm{Q}^{+} / \mathrm{N}^{-}\right.$or $\left.\mathrm{Q}^{-} / \mathrm{N}^{+}\right)$before the addition of the reagents. After stirring the reagents in both chambers, the current recording was started immediately at the voltage that exhibited the Q-trace, because the difference in gating between S-S and SH-SH is more obvious in the Q-traces (Fig. 3). OmpG often closes at high potentials (>100mV); ${ }^{[24,30]}$ therefore, \pm 50 $\mathrm{mV}$ were applied throughout the assay to avoid closure of the pore. For a typical $\mathrm{Q}^{+} / \mathrm{N}^{-}$ pore, the addition of DTT to the cis chamber and $\mathrm{H}_{2} \mathrm{O}_{2}$ to the trans chamber altered the gating behavior of the pore; $P_{\text {gating }}$ increased from a value of 0.013 to 0.055 . Moreover, the amplitudes of the gating events shifted to a higher value (Fig. 5B). The changes in $P_{\text {gating }}$ and the events amplitude distribution indicate that DTT had cleaved the disulfide bond and the S-S pore had converted to a SH-SH pore. Importantly, this result also suggests that the disulfide bond of the $\mathrm{Q}^{+} / \mathrm{N}^{-}$pore was located in the cis chamber in this experiment. For a different $\mathrm{Q}^{+} / \mathrm{N}^{-}$pore, the addition of DTT to the trans chamber caused only a slight increase in $P_{\text {gating }}$ (Fig. 5C). In this case, the events amplitude distribution did not change after the addition of the reagents, suggesting that the disulfide bond remained intact (Fig. 5C). Therefore, in the case of the $\mathrm{Q}^{+} / \mathrm{N}^{-}$pore, we deduce that the disulfide bond is exposed in the cis chamber. By contrast, with $\mathrm{Q}^{-} / \mathrm{N}^{+}$pores, the addition of DTT to the cis chamber had no effect (Fig. 5D), while DTT in the trans chamber changed the quiet pore into a noisier pore with high-amplitude gating events (Fig. 5E). We deduce that the disulfide bond of the $\mathrm{Q}^{-} / \mathrm{N}^{+}$ pores is exposed in the trans chamber. In summary, the extracellular loops of $\mathrm{Q}^{+} / \mathrm{N}^{-}$pores are located in the cis chamber, and for $\mathrm{Q}^{-} / \mathrm{N}^{+}$pores they are in the trans chamber.

\section{Binding of $a_{7} f \beta C D$ to $q O m p G$ from the extracellular and periplasmic entrances}

In stochastic sensing, molecular adapters can be used to provide binding sites for guest analytes. We have developed previously an OmpG pore engineered for reduced gating activity, the "quiet" OmpG (qOmpG) pore, which is the OmpG S-S mutant with an additional Asp-215 deletion. ${ }^{[29]}$ Since the asymmetry in the gating pattern and unitary conductance values is also preserved in this pore (Fig. S2), the orientation of qOmpG pores can be deduced according to the orientation rule revealed earlier and then used to investigate the interaction of an adapter with respect to the sideness of addition.

First, we examined qOmpG exposed to heptakis-(6-deoxy-6-amino)- $\beta$-cyclodextrin $\left(\mathrm{am}_{7} \beta \mathrm{CD}\right)$ from the periplasmic side (Fig. 6A). As a typical example, here we show a $\mathrm{Q}^{-} / \mathrm{N}^{+}$ pore (trans orientation). The current was recorded to display the background gating (Fig. 6B, top). $\mathrm{Am}_{7} \beta \mathrm{CD}$ was then added to the cis chamber. At a potential of $-50 \mathrm{mV}, \mathrm{am}_{7} \beta \mathrm{CD}$ caused transient current blockades that were visible on top of the gating events (Fig. 6B, top). Event distribution plots revealed that the $\mathrm{am}_{7} \beta \mathrm{CD}$ binding events comprise a single population featuring an $\sim 80 \%$ current block (Fig. $6 \mathrm{C}$, top), suggesting that there is only one binding site for $\mathrm{am}_{7} \beta C D$ in the $\mathrm{qOmpG}$ pore. Dwell time histograms of the $\mathrm{am}_{7} \beta \mathrm{CD}$ events could be fitted with single exponential functions that yielded a mean dwell time of $0.24 \pm$ $0.06(n=3) \mathrm{ms}$. The background gating events are mostly located in a region of the event distribution plot with a dwell time of $<0.1 \mathrm{~ms}$ and a current block of $<40 \%$. A more negative potential stimulates the occurrence of the $\mathrm{am}_{7} \beta \mathrm{CD}$ binding events (the population with $80 \%$ current block) (Fig. 6D, left). Furthermore, the mean dwell time of $\mathrm{am}_{7} \beta \mathrm{CD}$ in the qOmpG pore shows an exponential increase with increasing voltage as expected (Fig. 6E). ${ }^{[31]}$

In separate experiments, we studied the binding of $\mathrm{am}_{7} \beta \mathrm{CD}$ to $\mathrm{qOmpG}$ from the extracellular side. As an example, a $\mathrm{Q}^{+} / \mathrm{N}^{-}$pore in the cis orientation is shown (Fig. $6 \mathrm{~A}$, bottom). After recording the background gating, $\mathrm{am}_{7} \beta \mathrm{CD}$ was added to the cis chamber and 
a potential of $-50 \mathrm{mV}$ was applied, which produced numerous transient current blockades (Fig. 6B, bottom). Event distribution plots show that the new events are of low amplitude $(<50 \%$ block) and mostly scattered around the zone with short dwell times $(<0.1 \mathrm{~ms})$ (Fig. $6 \mathrm{C}$, bottom). These events do not form a single defined population suggesting that at moderate potentials qOmpG provides multiple weak binding sites for $\mathrm{am}_{7} \beta C D$ entering through the extracellular end of the pore. At high applied potentials $(>75 \mathrm{mV})$, a new population featuring a $95 \%$ current block becomes equally dominant (Fig. 6D, right). The mean dwell time of this population increases slowly with the applied potential (Fig. 6E). The population of events with the characteristic $80 \%$ current block seen when $a_{7} \beta C D$ binds from the periplasmic side does not appear when $\mathrm{am}_{7} \beta \mathrm{CD}$ is presented to the extracellular side of OmpG. This suggests that $a_{7} \beta C D$ delivered from the periplasmic side of the $q O m p G$ pore does not bind at the same site as $a_{7} \beta C D$ delivered from the extracellular side.

\section{Conclusion}

We have shown that the gating behavior and conductance of the OmpG pore are asymmetric with respect to the applied potential. Further, the absolute orientation of individual pores can be established from the sidedness of the response of an extracellular disulfide bond to the reducing agent DTT. By these means, the connection between the electrical asymmetry and the orientation of OmpG has been firmly established. Based on this work, electrical measurements alone can now be used to determine the orientation of the OmpG pore.

The OmpG pore is inserted into planar bilayers by detergent dilution, an approach we and others have used with several proteins over many years. ${ }^{[24,25,32,33]}$ Correctly folded OmpG in $n$-dodecyl $\beta$-D-maltoside (DDM) or $n$-octyl $\beta$-D-glucopyranoside (OG) at above the critical micelle concentration is diluted into the cis chamber of the bilayer apparatus. The subsequent insertion is desirably inefficient; after all, the approach has been developed for single-channel recording. Consequently, the mechanism by which OmpG inserts into lipid bilayers after detergent dilution remains unclear. Presumably, the protein retains a ring of detergent that dissociates during insertion, becoming sufficiently diluted that the bilayer is not perturbed. Considering the asymmetric structure of OmpG, it is surprising that it inserts into the lipid bilayer with little preference for a particular orientation. In particular, when the pore adopts the trans orientation, the extracellular loops must penetrate through the bilayer. Since the extracellular loops contain 21 negatively and 4 positively charged residues in total (Fig. S3), such a process is energetically unfavorable. By contrast, insertion of the trimeric maltoporin was reported to be largely unidirectional, with $95 \%$ of the pores inserting into the lipid bilayer with their short turns first. ${ }^{[18]}$ In the structure of the trimeric maltoporin from E. coli (pdb code: 1MAL), three extracellular loops (L1, L3 and L5) from each monomer are folded inside the barrel lumen, while the additional five loops are exposed to the extracellular environment. ${ }^{[34]}$ These five loops carry $\sim 29$ charges ( $\sim 20$ negative and $\sim 9$ positive). As a result, a trimer has $\sim 87$ charged residues at the extracellular end of the barrel. Thus, the difference in the distribution of orientations between maltoporin and OmpG may be due to the greater number of charged residues on the extracellular loops of the trimer as compared to the monomer. Nevertheless, questions remain concerning the means by which the charged loops of OmpG penetrate spontaneously into the lipid bilayer. Several molecule dynamics (MD) simulations provide some clues about this issue. A coarse grain model of a hydrophobic hollow tube with two hydrophilic termini was constructed as a representation of a transmembrane pore. ${ }^{[35,36]}$ The simulations show that the model pore can spontaneously insert into a 1,2-dimyristoyl-sn-glycero-3-phosphocholine (DMPC) lipid bilayer. Most interestingly, two lipid molecules from the leaflet on which the pore docks form salt bridges with a hydrophilic end of the pore and assist its crossing of the bilayer. Therefore, we speculate that the lipids and/or ions from solution might be involved in 
shielding the charges of the extracellular loops of OmpG and thereby aid penetration through the bilayer.

In contrast with the present study, we have also found that OmpG pores always insert with the short periplasmic turns first into lipid bilayers formed between aqueous droplets in oil. ${ }^{[30]}$ Further, this unidirectional insertion is independent of the charge on the headgroup of the bilayer. ${ }^{[30]}$ Therefore, we propose that the physical properties of the bilayer, e.g. bilayer elasticity, play a more important role than the chemical constitution of the lipids in determining the direction of protein insertion. In bacterial cells, all outer membrane porins reside with their long loops in an extracellular orientation. It remains unclear whether in vivo such an insertion process occurs spontaneously from the periplasmic compartment, or is under the regulation of cellular chaperones. ${ }^{[37,38]}$

We also examined the binding of a molecular adapter, $a_{7} \beta C D$, to $q O m p G$ and observed differences that depended on whether the adapter binds from the periplasmic or the extracellular entrance to the pore. OmpG has a relatively wide entrance at its periplasmic end, which opens up to more than $15 \AA$ in internal diameter. ${ }^{[29]}$ The tunnel through the lumen of the pore narrows down to $8 \AA$ in diameter near the extracellular end. By comparison, the $a_{7} \beta C D$ ring has an outer diameter of $15 \AA$, ${ }^{[39]}$ which, taking into account the flexibility of the $\beta$ barrel structure, would allow it to enter the OmpG pore from the periplasmic end. However, the depth to which the $a_{7} \beta C D$ molecule can travel towards the extracellular end of the pore is limited by steric hindrance. Therefore, under an applied potential, $\mathrm{am}_{7} \beta \mathrm{CD}$ can either lodge within the pore or escape by exiting back through the periplasmic entrance. The exponential increase in the dwell time of the blocking events with increasing applied potential supports this hypothesis. By comparison, $a_{7} \beta C D$ presented from the extracellular side is not able to enter the lumen. In this case, the transient associations that are observed between $\mathrm{am}_{7} \beta \mathrm{CD}$ and the $\mathrm{qOmpG}$ protein are most likely mediated by electrostatic interactions between the positively charged $\mathrm{am}_{7} \beta C D$ and the highly negatively charged $\mathrm{qOmpG}$ loops. $\mathrm{Am}_{7} \beta \mathrm{CD}$ may bind either to one of the loops or to several loops simultaneously, leading to events of different amplitudes. Since $\mathrm{am}_{7} \beta \mathrm{CD}$ is not confined within the pore lumen, it can readily dissociate back into the bulk solution, which might explain the relatively short dwell time and low affinity when it is presented from the extracellular side.

For applications in stochastic sensing, it is important that the dwell time of an adapter within the lumen of the sensor pore is as long as possible and that there is enough current remaining after the adapter binds for detection of an analyte that in turn binds within the adapter. ${ }^{\text {[31] }}$ The information on the interaction between the $\mathrm{am}_{7} \beta \mathrm{CD}$ and $\mathrm{qOmpG}$ serves to advance the use of qOmpG as a biosensor. As demonstrated above, the binding of $\mathrm{am}_{7} \beta \mathrm{CD}$ at either the extracellular or periplasmic sides qOmpG modulates the ionic current flowing through the pore. However, $a_{7} \beta C D$ presented from the extracellular side cannot serve as a useful molecule adapter; the binding interactions with qOmpG are either too short or there is insufficient remaining current. By contrast, $a_{7} \beta C D$ presented from the periplasmic side produces longer binding events, and the remaining current is large enough to allow the detection of the binding of a second molecule within the cyclodextrin ring. [29]

\section{Experimental Section}

\section{Mutagenesis, expression and purification of OmpG}

The pT7-OmpG plasmids used in this work were constructed previously ${ }^{[29]}$ and transformed into E. coli PC2889 cells [BL21(DE3) $\Delta$ lamB ompR]. Cells were grown in LB medium at $37^{\circ} \mathrm{C}$ until the $\mathrm{OD}_{600}$ reached 1.0 , when IPTG $(0.5 \mathrm{mM}$, final concentration) was added and the cells cultured for a further $3 \mathrm{~h}$ before harvesting. Pellets from the culture $(0.5$ 
L) were resuspended in Tris. $\mathrm{HCl}$ buffer $(50 \mathrm{mM}, \mathrm{pH} 8.0,30 \mathrm{~mL}$ ) containing lysozyme (200 $\mu \mathrm{g} / \mathrm{mL}$ ) and EDTA $(1 \mathrm{mM})$, and incubated at room temperature for $30 \mathrm{~min}$. DNase I ( $5 \mu \mathrm{L}$, $2,000 \mathrm{U} / \mu \mathrm{L})$, with $\mathrm{MgCl}_{2}$ ( $2 \mathrm{mM}$, final concentration), was added to the mixture to decrease the viscosity. The lysate was centrifuged at $22,300 \mathrm{xg}$ for $30 \mathrm{~min}$. The pellet containing OmpG was washed once with buffer (30 mL $50 \mathrm{mM}$ Tris. $\mathrm{HCl}, \mathrm{pH} 8.0,1.5 \mathrm{M}$ urea), dissolved in denaturation buffer (50 mM Tris. $\mathrm{HCl}, \mathrm{pH} 8.0,8 \mathrm{M}$ urea, $50 \mathrm{~mL}$ ) and passed through a $0.22 \mu \mathrm{m}$ filter before FPLC separation.

\section{Refolding of OmpG}

The OmpG extract was loaded onto a Q-Sepharose column $(10 \mathrm{~mL})$ and eluted with a 0 to $0.5 \mathrm{M} \mathrm{NaCl}$ gradient over $30 \mathrm{~min}$ in denaturation buffer. The purified OmpG was refolded by dilution with refolding buffer $(20 \mathrm{mM}$ Tris.HCl, $\mathrm{pH}$ 9.0, 3.25\% OG (n-octyl- $\beta$-Dglucopyranoside)) until the final urea concentration reached 3.0 M. In the case of the cysteine mutant, the refolding buffer also contained DTT $(10 \mathrm{mM})$ to prevent the formation of intermolecular disulfide bonds. The refolding solution was incubated at $37^{\circ} \mathrm{C}$ over $48 \mathrm{~h}$ and the refolding efficiency was determined by SDS-PAGE analysis. ${ }^{28,29]}$

\section{Formation of the disulfide bond}

To form the disulfide bridge within the OmpG cysteine mutant, DTT was removed from the refolded sample with a desalting column equilibrated with Tris. $\mathrm{HCl}$ buffer $(50 \mathrm{mM}, \mathrm{pH} 8.0)$ containing DDM (n-dodecyl $\beta$-D-maltoside) $(0.008 \% \text {, w/v). Cu (o-phenanthroline) })_{2}$ (prepared as a mixture of $\mathrm{CuSO}_{4}$ and o-phenanthroline in a ratio of $1: 3.5 \mathrm{~mol} / \mathrm{mol}$ ) was added to the protein solution to a final concentration of $1.3 \mathrm{mM}$. After $5 \mathrm{~min}$ at room temperature, EDTA ( $5 \mathrm{mM}$, final concentration) was added to stop the reaction. The sample was then applied to a Centricon size-exclusion filter with a $10 \mathrm{kDa}$ cut off (Fisher Scientific, $\mathrm{UK})$ to remove the excess reagents and exchange the buffer into Tris. $\mathrm{HCl}$ buffer $(50 \mathrm{mM}$, $\mathrm{pH}$ 8.0) containing DDM $(0.004 \%, \mathrm{w} / \mathrm{v})$.

\section{Single channel recordings of OmpG}

Planar lipid bilayer experiments were performed in an apparatus partitioned into two chambers with a $25 \mu \mathrm{m}$-thick Teflon film. An aperture of approximately $100-\mu \mathrm{m}$ diameter had been made near the center of the film with an electric arc. Each chamber was filled with Tris. $\mathrm{HCl}(10 \mathrm{mM}, \mathrm{pH} 8.5)$ and $\mathrm{KCl}(1 \mathrm{M})$. A Ag/ $\mathrm{AgCl}$ electrode was immersed in each chamber with the cis chamber grounded. A positive potential indicates a higher potential in the trans chamber. 1,2-Diphytanoyl-sn-glycerol-3-phosphocholine (Avanti Polar Lipids, USA) dissolved in pentane (10\% v/v) was deposited on the surface of the buffer in both chambers and monolayers formed after the pentane evaporated. The lipid bilayer was formed by raising the liquid level up and down across the aperture, which had been pretreated with a hexadecane/pentane (1:10 v/v) solution. OmpG protein ( 1 to $5 \mu l$ of $\sim 0.5$ $\mathrm{mg} \mathrm{mL}^{-1}$ ) was added to the cis chamber and then a potential of $+200 \mathrm{mV}$ was applied to induce protein insertion. After a single channel had inserted into the bilayer, the ionic current was recorded at $\pm 50 \mathrm{mV}$, unless otherwise stated. The current was amplified with an Axopatch 200B integrating patch clamp amplifier (Axon Instruments, Foster City, CA). Signals were filtered with a Bessel filter at $2 \mathrm{kHz}$ (unless otherwise stated) and then acquired by a computer (sampling at $50 \mu \mathrm{s}$ ) after digitization with a Digidata 1320A/D board (Axon Instruments). Data were analyzed with Clampex 10.0 software.

\section{Supplementary Material}

Refer to Web version on PubMed Central for supplementary material. 


\section{Acknowledgments}

We thank Dr. Matthew Holden for his comments on the manuscript. This work was supported by grants from the MRC and the NIH. HB is the holder of a Royal Society Wolfson Research Merit Award.

\section{References}

1. Tien, HT.; Ottova, A. Planar lipid bilayers(BLM'S) and their applications. Elsevier; 2003. p. 347-734.

2. Bayley H, Cremer PS. Nature. 2001; 413:226-230. [PubMed: 11557992]

3. Schmidt J. Journal of Materials Chemistry. 2005; 15:831-840.

4. Delcour AH. J Mol Microbiol Biotechnol. 2002; 4:1-10. [PubMed: 11763966]

5. Basle A, Iyer R, Delcour AH. Biochim Biophys Acta. 2004; 1664:100-107. [PubMed: 15238263]

6. Zakharov SD, Eroukova VY, Rokitskaya TI, Zhalnina MV, Sharma O, Loll PJ, Zgurskaya HI, Antonenko YN, Cramer WA. Biophys J. 2004; 87:3901-3911. [PubMed: 15465872]

7. Liu N, Delcour AH. Protein Eng. 1998; 11:797-802. [PubMed: 9796829]

8. Basle A, Qutub R, Mehrazin M, Wibbenmeyer J, Delcour AH. Protein Eng Des Sel. 2004; 17:665672. [PubMed: 15469993]

9. Sheppard DN, Gray MA, Gong X, Sohma Y, Kogan I, Benos DJ, Scott-Ward TS, Chen JH, Li H, Cai Z, Gupta J, Li C, Ramjeesingh M, Berdiev BK, Ismailov, Bear CE, Hwang TC, Linsdell P, Hug MJ. J Cyst Fibros. 2004; 3(Suppl 2):101-108. [PubMed: 15463939]

10. Hill K, Model K, Ryan MT, Dietmeier K, Martin F, Wagner R, Pfanner N. Nature. 1998; 395:516521. [PubMed: 9774109]

11. Nassi S, Collier RJ, Finkelstein A. Biochemistry. 2002; 41:1445-1450. [PubMed: 11814336]

12. Bayley H, Jayasinghe L. Mol Membr Biol. 2004; 21:209-220. [PubMed: 15371010]

13. Song L, Hobaugh MR, Shustak C, Cheley S, Bayley H, Gouaux JE. Science. 1996; 274:1859_ 1866. [PubMed: 8943190]

14. Koronakis V, Sharff A, Koronakis E, Luisi B, Hughes C. Nature. 2000; 405:914-919. [PubMed: 10879525]

15. Andersen C, Hughes C, Koronakis V. J Membr Biol. 2002; 185:83-92. [PubMed: 11891567]

16. Heginbotham L, LeMasurier M, Kolmakova-Partensky L, Miller C. J Gen Physiol. 1999; 114:551560. [PubMed: 10498673]

17. Kutluay E, Roux B, Heginbotham L. Biophys J. 2005; 88:1018-1029. [PubMed: 15556975]

18. Danelon C, Brando T, Winterhalter M. J Biol Chem. 2003; 278:35542-35551. [PubMed: 12835320]

19. Terstappen GC. Drug Discovery Today: Technologies. 2005; 2:133.

20. Dunlop J, Bowlby M, Peri R, Vasilyev D, Arias R. Nat Rev Drug Discov. 2008; 7:358-368. [PubMed: 18356919]

21. Gu LQ, Braha O, Conlan S, Cheley S, Bayley H. Nature. 1999; 398:686-690. [PubMed: 10227291]

22. Cheley S, Gu LQ, Bayley H. Chem Biol. 2002; 9:829-838. [PubMed: 12144927]

23. Fajardo DA, Cheung J, Ito C, Sugawara E, Nikaido H, Misra R. J Bacteriol. 1998; 180:4452-4459. [PubMed: 9721282]

24. Conlan S, Zhang Y, Cheley S, Bayley H. Biochemistry. 2000; 39:11845-11854. [PubMed: 11009596]

25. Conlan S, Bayley H. Biochemistry. 2003; 42:9453-9465. [PubMed: 12899633]

26. Yildiz O, Vinothkumar KR, Goswami P, Kuhlbrandt W. EMBO J. 2006; 25:3702-3713. [PubMed: 16888630]

27. Subbarao GV, van den Berg B. J Mol Biol. 2006; 360:750-759. [PubMed: 16797588]

28. Liang B, Tamm LK. Proc Natl Acad Sci U S A. 2007; 104:16140-16145. [PubMed: 17911261]

29. Chen M, Khalid S, Sansom MS, Bayley H. Proc Natl Acad Sci U S A. 2008; 105:6272-6277.

[PubMed: 18443290] 
30. Hwang WL, Chen M, Cronin B, Holden MA, Bayley H. J Am Chem Soc. 2008; 130:5878-5879. [PubMed: 18407631]

31. Gu LQ, Cheley S, Bayley H. J Gen Physiol. 2001; 118:481-494. [PubMed: 11696607]

32. Fischer K, Weber A, Brink S, Arbinger B, Schunemann D, Borchert S, Heldt HW, Popp B, Benz R, Link TA, et al. J Biol Chem. 1994; 269:25754-25760. [PubMed: 7523392]

33. Bishop ND, Lea EJ, Mobasheri H, Spiro S. FEBS Lett. 1996; 379:295-298. [PubMed: 8603709]

34. Schirmer T, Keller TA, Wang YF, Rosenbusch JP. Science. 1995; 267:512-514. [PubMed: 7824948]

35. Lopez CF, Nielsen SO, Moore PB, Klein ML. Proc Natl Acad Sci U S A. 2004; 101:4431-4434. [PubMed: 15070735]

36. Lopez CF, Nielsen SO, Ensing B, Moore PB, Klein ML. Biophys J. 2005; 88:3083-3094. [PubMed: 15722425]

37. Tommassen J. Science. 2007; 317:903-904. [PubMed: 17702930]

38. Bos MP, Robert V, Tommassen J. Annu Rev Microbiol. 2007; 61:191-214. [PubMed: 17506684]

39. Schurig V, Nowotny HP. Angewandte Chemie. 1990; 102:969-986.Angewandte Chemie International Edition in English. 1990; 29:939-957. 


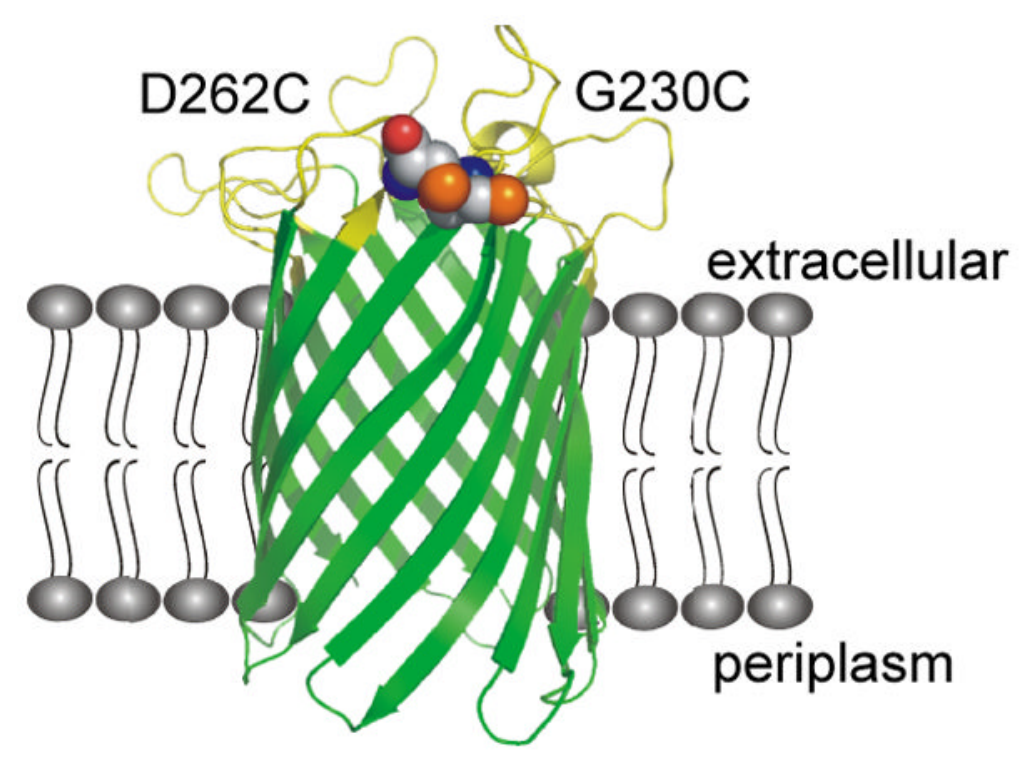

Figure 1. Structure of OmpG G230C/D262C

The model of OmpG G230C/D262C was created in Pymol based on the structure of OmpG in an open conformation (PDB code: 2IWV). Cys-230 and Cys-262 are highlighted as sticks and balls. The large loops in yellow are located at the extracellular entrance to the pore, while the short turns face the periplasm. 

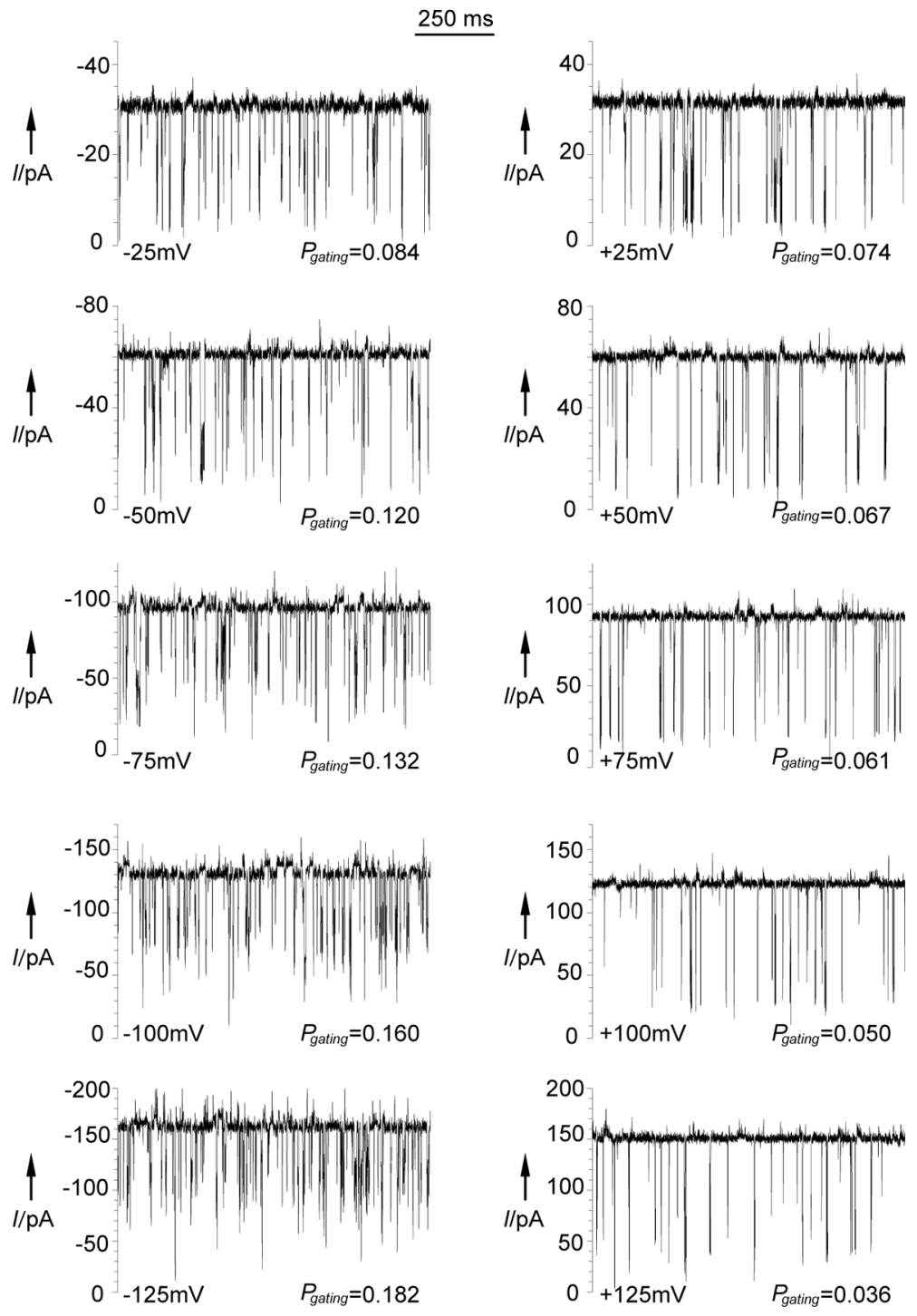

Figure 2. Single channel recordings from WT OmpG

Current traces (1 s) from a typical WT OmpG pore at various applied potentials. The buffer was $10 \mathrm{mM}$ Tris. $\mathrm{HCl}, \mathrm{pH} 8.5,1 \mathrm{M} \mathrm{KCl}$. The $P_{\text {gating }}$ values are the time a pore resides in a closed (zero current) or partially closed state (current smaller than that of the fully open state) divided by the total recording time. The pore exhibits an asymmetric gating pattern. As indicated by the $P_{\text {gating }}$ values, the gating activity at a negative potential is higher than that at a positive one. The differences between the two traces are enlarged at high potentials: the positive currents become quieter, while the negative currents become noisier. 
A) S-S

$250 \mathrm{~ms}$
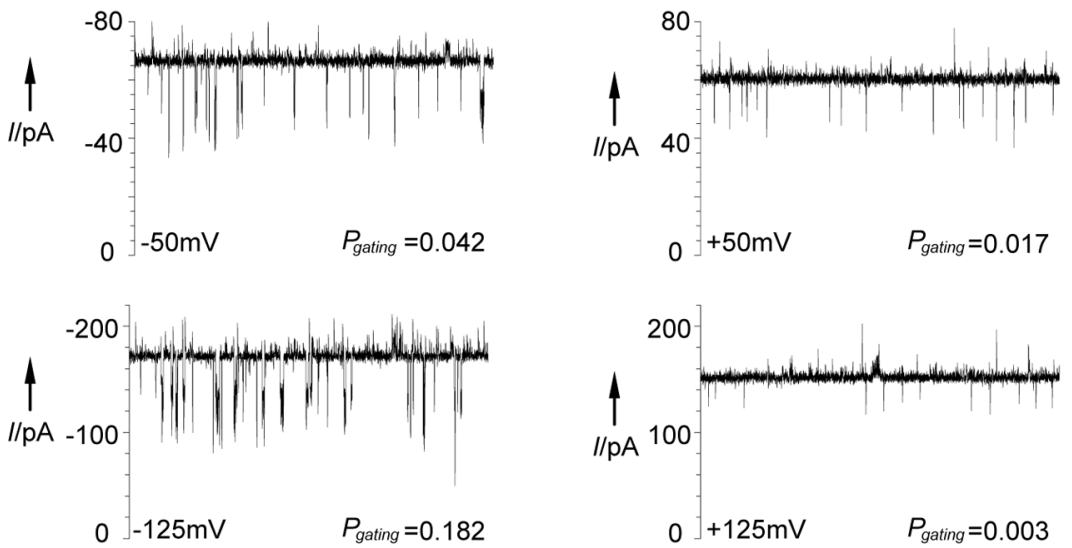

B)

SH-SH
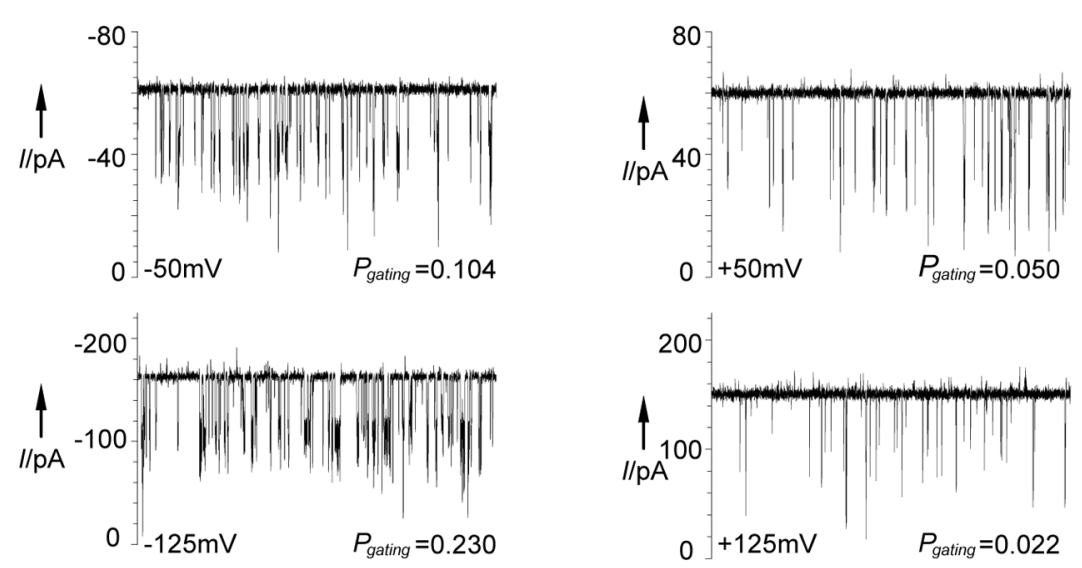

C)
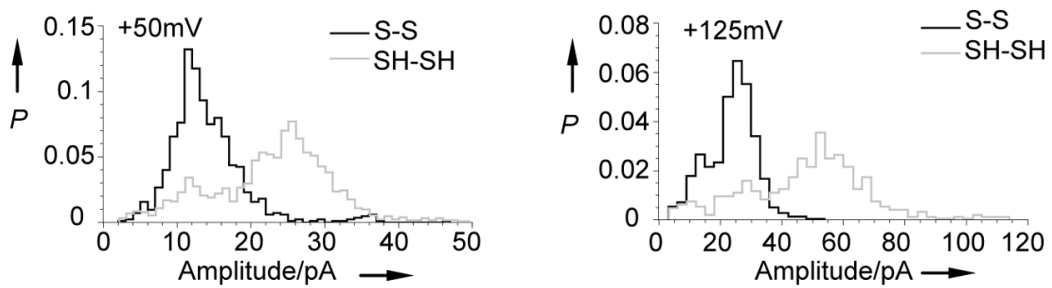

Figure 3. Single channel recordings from OmpG S-S and SH-SH

Typical examples of Q-traces $(1 \mathrm{~s})$ for each mutant at various applied potentials in the $\mathrm{Q}^{+}$/ $\mathrm{N}^{-}$orientation are displayed. Each chamber contained $10 \mathrm{mM}$ Tris. $\mathrm{HCl}, \mathrm{pH} 8.5,1 \mathrm{M} \mathrm{KCl}$ in the absence (A: S-S) or presence (B: SH-SH) of $10 \mathrm{mM}$ DTT. To obtain SH-SH, OmpG S-S was incubated with $20 \mathrm{mM}$ DTT for $30 \mathrm{~min}$ in $50 \mathrm{mM}$ Tris. $\mathrm{HCl}, \mathrm{pH} 8.5,0.004 \%$ DDM before addition to the chamber. (C): Comparison of the event amplitude histograms derived from the Q-traces of S-S and SH-SH. The applied potentials were $+50 \mathrm{mV}$ (left) and +125 $\mathrm{mV}$ (right). P: probability. 

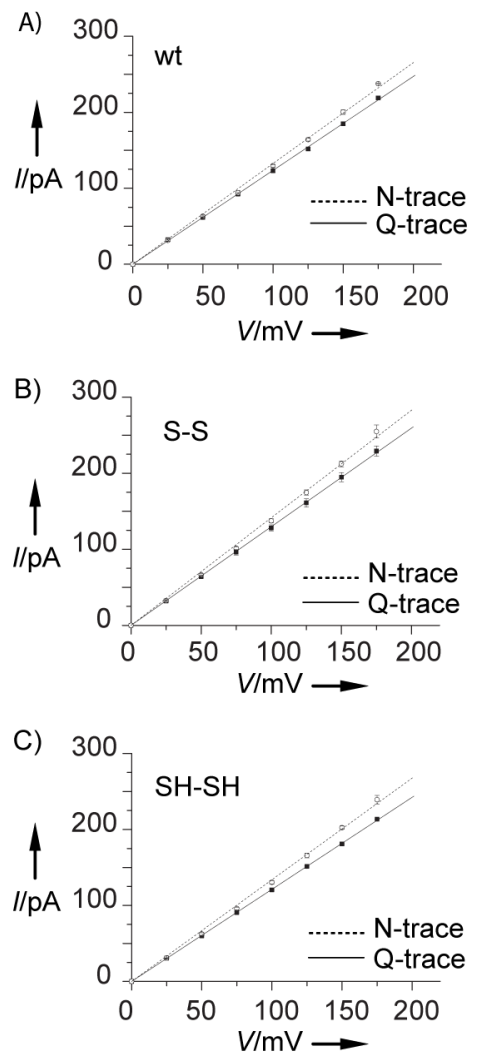

Figure 4. I-V curves of the Q-traces and N-traces of OmpG proteins

Current-voltage relationships of Q-traces (solid lines) and N-traces (dashed lines) are shown. The buffer used was $10 \mathrm{mM}$ Tris. $\mathrm{HCl}, \mathrm{pH} 8.5,1 \mathrm{M} \mathrm{KCl}$. The data represent the mean values from three independent pores. The bars show the standard deviations. 
A)
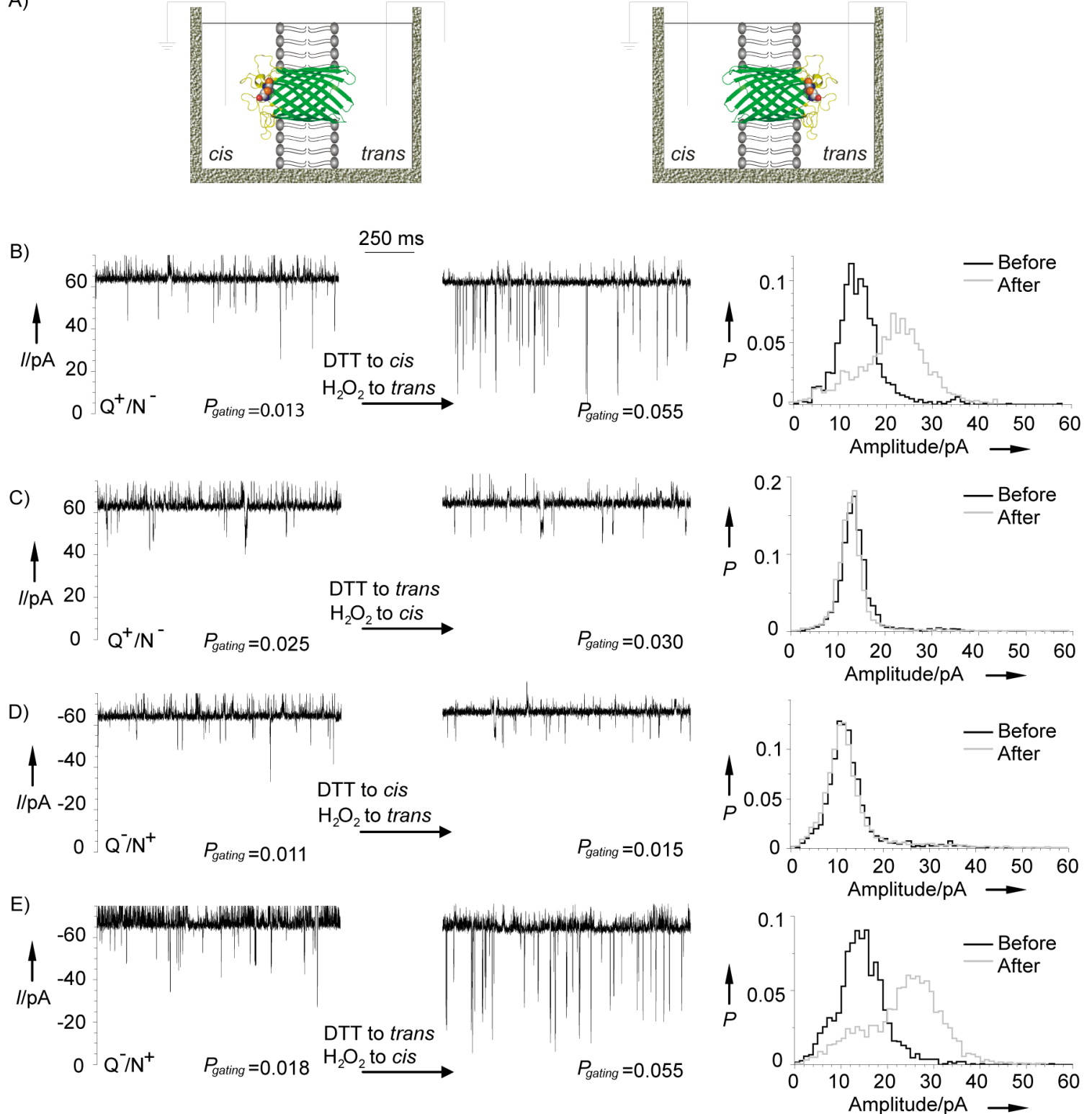

Figure 5. Location of the disulfide bond in the S-S pore

(A) The orientation of the OmpG S-S pore. Left: the orientation of OmpG S-S defined as cis. The extracellular aspect of the protein is located in the cis chamber. Right: the trans orientation of OmpG S-S. The extracellular aspect of the protein is located in the trans chamber. (B, C, D, E) the effect of DTT and $\mathrm{H}_{2} \mathrm{O}_{2}$ on the gating behavior of the pores. Each

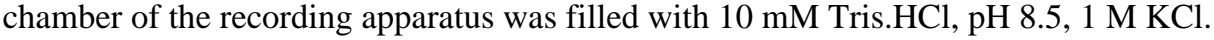
OmpG S-S was added to the cis chamber which was grounded. After a single S-S pore had inserted, the current was recorded at $\pm 50 \mathrm{mV}$ for $5 \mathrm{~min}$. The potential was switched to 0 $\mathrm{mV}$, and $2.0 \mathrm{M}$ DTT $(10 \mu \mathrm{L})$ and $30 \%(\mathrm{v} / \mathrm{v}) \mathrm{H}_{2} \mathrm{O}_{2}(10 \mu \mathrm{L})$ were added to the chambers $(1$ $\mathrm{mL} / \mathrm{chamber}$ ) as indicated. Both chambers were immediately stirred for $10 \mathrm{~s}$ and the potential was switched back to $+50 \mathrm{mV}$ or $-50 \mathrm{mV}$ depending on which potential gave the Q-trace. Left: The Q-trace of the current recording (1 s) of a single $\mathrm{S}$-S pore. The relative orientation of each pore $\left(\mathrm{Q}^{+} / \mathrm{N}^{-}\right.$or $\left.\mathrm{Q}^{-} / \mathrm{N}^{+}\right)$is indicated. Middle: current recording $(1 \mathrm{~s})$ after the reagents were added to the pore shown to the left. The chambers to which the reagents 
were added is indicated above the arrows. The $P_{\text {gating }}$ values of the Q-trace before and after the reagents were added are also shown. Right: the events amplitude histogram before and after the addition of reagents. Before: before the reagents; After: after the reagents. P: probability. 
A)

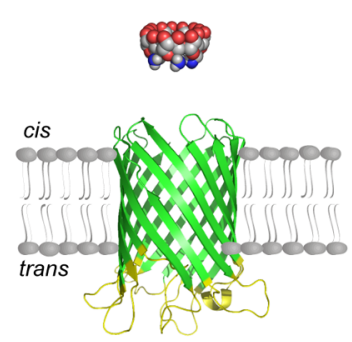

D)

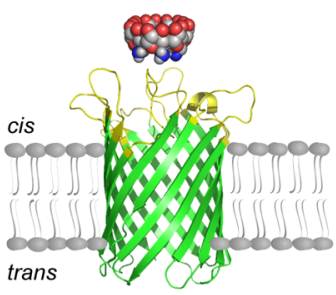

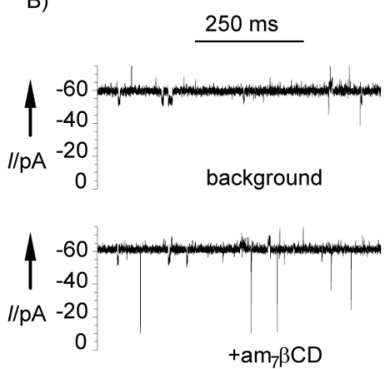
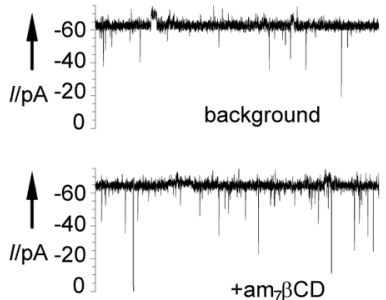

C)

event density $(\mathrm{N} / \log (\mathrm{ms}))$
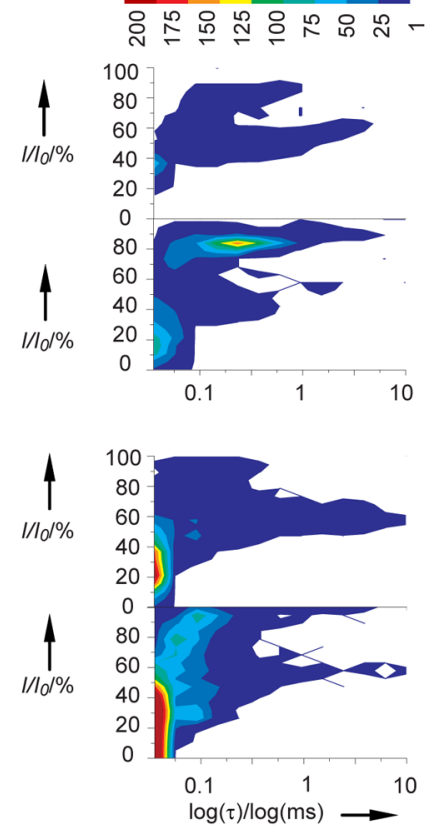

E)
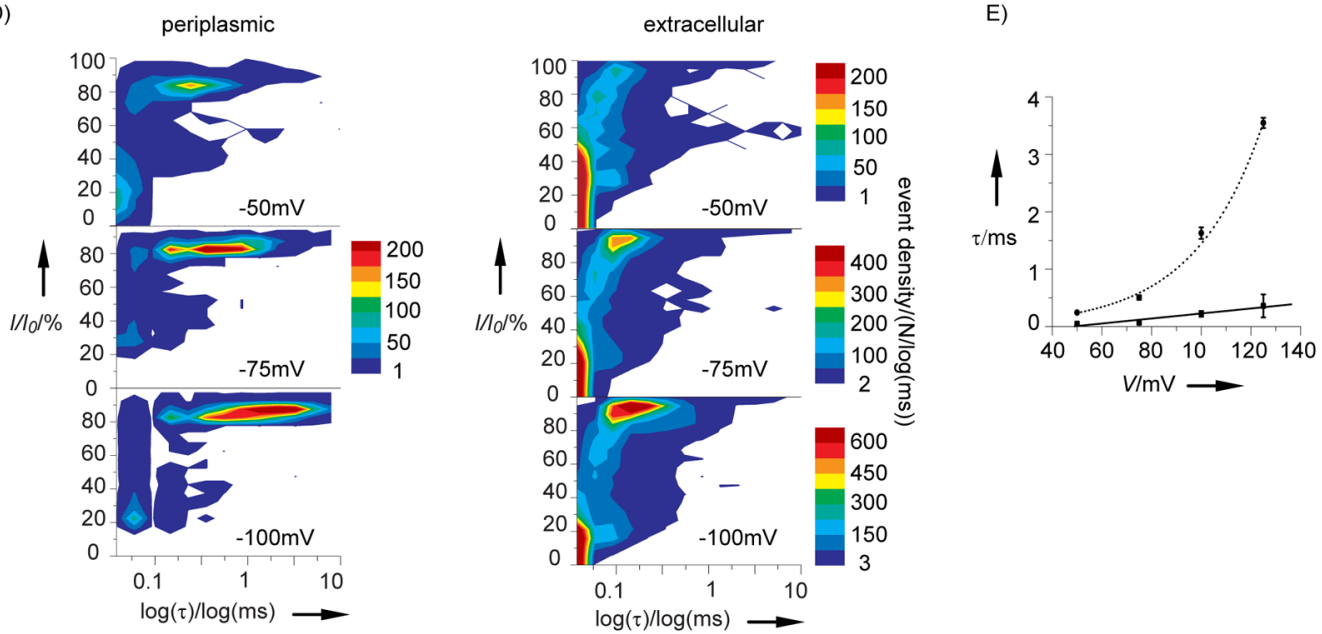

Figure 6. Interactions of $q \mathrm{OmpG}$ with am7f $\beta \mathrm{CD}$ applied from the periplasmic or the extracellular side

(A) Cartoon representation of $a_{7} \beta C D$ approaching the $q O m p G$ pore from the periplasmic side (top) or the extracellular side (bottom). (B) Current recording of a single qOmpG pore in the trans (top) or cis (bottom) orientation in the absence (background) and presence

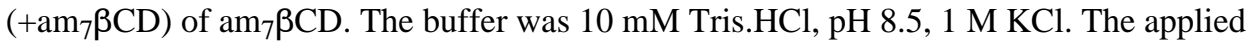
potential was $-50 \mathrm{mV}$. $\mathrm{Am}_{7} \beta \mathrm{CD}$ was added to the cis chamber to a final concentration of $0.5 \mu \mathrm{M}$. Signals were processed at $10 \mathrm{kHz}$ with a Bessel filter and acquired at a sampling rate of $50 \mathrm{kHz}$. For display, the traces were filtered with a $2 \mathrm{kHz}$ Gaussian filter. Top: periplasmic binding; bottom: extracellular binding. (C) Event distribution plots corresponding to traces in (B). The distribution of gating events for $100 \mathrm{~s}$ of recording is plotted according to the event amplitudes and dwell times. The density of events is indicated 
by the color code. (D) Event distribution plots from traces in the presence of $\mathrm{am}_{7} \beta \mathrm{CD}$ at increasing applied potentials. Because of the drastically increased number of events at high voltages, the color code was adjusted for the events arising from extracellular addition to optimally reveal the distribution patterns. (E) Effect of the applied potential on the mean dwell time. Dashed line: $a_{7} \beta C D$ binding from the periplasmic side. The dwell time histograms for the $80 \%$-blockade events at various applied potentials were fitted with single exponential functions and the mean dwell times were derived. The mean dwell times were plotted against the applied potential and the plot fitted to a single exponential function. Solid line: $\mathrm{am}_{7} \beta \mathrm{CD}$ binding from the extracellular side. The dwell time histograms for the $95 \%$ blockade events were fitted with single exponential functions and the mean dwell times derived. The relationship between the mean dwell times and the applied potentials were fitted with a single exponential function. The bars indicate the standard deviations from three independent measurements. $\tau$ : dwell time. 